Revue d'Allemagne et des pays de langue allemande

46-1| 2014

Les fondements normatifs de l'État constitutionnel moderne en Allemagne. Une approche pluridisciplinaire

\title{
La démocratie chrétienne, de la victoire à la grande coalition
}

Jean-Louis Georget

\section{QpenEdition \\ Journals}

Édition électronique

URL : https://journals.openedition.org/allemagne/1407

DOI : $10.4000 /$ allemagne. 1407

ISSN : 2605-7913

Éditeur

Société d'études allemandes

Édition imprimée

Date de publication : 30 juin 2014

Pagination : 171-183

ISSN : 0035-0974

Référence électronique

Jean-Louis Georget, «La démocratie chrétienne, de la victoire à la grande coalition », Revue

d'Allemagne et des pays de langue allemande [En ligne], 46-1 | 2014, mis en ligne le 29 juillet 2019,

consulté le 18 mai 2021. URL : http://journals.openedition.org/allemagne/1407 ; DOI : https://doi.org/ 10.4000/allemagne.1407 


\section{La démocratie chrétienne, de la victoire à la grande coalition}

- Jean-Louis Georget*

L'année 2013, qui s'est achevée avec le succès qu'on connaît pour la démocratie chrétienne, n’a pas été une année sans aspérités. Elle a commencé avec la déception d'une défaite électorale pour s'achever par une large victoire, due plus au tribut de la popularité de la personnalité de la chancelière qu'à la capacité de la démocratie chrétienne à envisager le modèle programmatique d'une société allemande en pleine mutation. À la manière dont ont évolué tous les partis, la CDU est devenue surtout une organisation capable d'emporter la décision politique dans les joutes électorales en conquérant de nouvelles circonscriptions sans perdre ses bastions. Lorsque c'est le cas, elle est capable de faire son aggiornamento et d'en tirer les conclusions pour prévenir toute débâcle. Elle s'est imposée comme l'axe autour duquel tourne désormais la vie politique allemande, ne se distinguant pas en termes d'orientation d'une Europe en crise qui se replie sur ses conservatismes.

\section{La leçon de la Basse-Saxe}

Le commencement de l'année électorale s'était distingué par de mauvais augures puisque les élections de Basse-Saxe, qui s'étaient tenues le 20 janvier 2013, s'étaient soldées contre toute probabilité, malgré la prudence des instituts de sondages, par une déroute cuisante pour la CDU de Hanovre, car elle avait essuyé une perte de 14 mandats par rapport aux élections précédentes, passant de $42 \%$ des suffrages obtenus en 2009 à $36 \%$. L’un des espoirs les plus en vue de la génération montante couvée par la chancelière, David MacAllister, avait été obligé de céder le pouvoir au profit d'une coalition social-démocrate et écologiste dirigée par Stephan Weil.

Contrairement à l'analyse de Bernd Ulrich, correspondant du magazine Die Zeit, qui avait attribué la défaite démocrate-chrétienne à une certaine volatilité de son

* Chercheur à l'Institut d'Histoire Français en Allemagne et membre du Centre Georg Simmel (UMR 8131 CNRS/EHESS). 
électorat ${ }^{(1)}$, on peut penser que les raisons étaient à chercher ailleurs. La campagne du candidat MacAllister avait été loin d'être flamboyante, comme le soulignait le journaliste Michael Inacker du Handelsblatt ${ }^{(2)}$, mais elle ne pouvait pas expliquer à elle seule l'évidence que la CDU n'avait pas su mobiliser ses électeurs. Outre le fait que la démocratie chrétienne avait pu toucher des publics électoraux qui n'étaient pas habituellement les siens, elle a réussi à rassembler ses électeurs autour de ses thèmes traditionnels et ses points forts comme la politique économique ( $46 \%$ contre 24 pour le SPD) et les finances (44\% contre 25 pour le SPD). Si des faux pas sont venus se glisser dans la campagne électorale comme l'inopportune introduction de droits estudiantins, renforçant l'idée que la CDU était toujours moins experte en matière d'éducation que le SPD, deux tiers des électeurs donnaient un satisfecit à l'action conduite par leur ministre-président.

La volonté de l'électorat était moins de voter en faveur du SPD que celle de marquer son insatisfaction envers la coalition avec le FDP. Les électeurs favorables à une telle constellation à $49 \%$ quatre ans auparavant n'étaient plus que $36 \%$ à donner leur blancseing à une telle association à la veille de l'élection du début de l'année 2013. Dans ce rejet se rejoignaient les images négatives du parti au niveau local, et particulièrement de son candidat Stefan Birkner, et celle qu'avait le public de la direction nationale et notamment de Philipp Rösler.

Sentant venir la défaite, les responsables de la CDU avaient appelé tacitement les électeurs à voter pour le FPD au moyen de leur second bulletin, permettant aux libéraux de conquérir 14 sièges, soit 9,9\% des voix, mais engendrant le déclin temporaire de la démocratie chrétienne dans la région. L'effet de vases communicants avait joué au détriment des chrétiens-démocrates. La stratégie suivie n'était pas efficace et, outre un acheminement évident vers la fin de la coalition conservatrice, Ronald Pofalla, secrétaire général de la CDU, en a tiré les conclusions pour les élections régionales et fédérales ultérieures.

\section{La CSU dominante en Bavière et le changement de situation en Hesse}

Les deux autres élections régionales de l'année 2013 en Bavière et en Hesse étaient presque simultanées avec les élections fédérales.

Malgré quelques accidents électoraux depuis sa création, la Bavière est souvent prévisible, tant la CSU y est hégémonique. Après quelques péripéties et grâce à Horst Seehofer, son président charismatique, elle a obtenu 101 mandats et 42,5\% des voix. La campagne a été menée tambour battant avec le résultat escompté, de sorte que la CSU a pesé de tout son poids sur les négociations de coalition à Berlin, prenant notamment ses distances envers une alliance possible avec le parti écologiste. Pour obtenir un score aussi probant, la CSU a rassemblé largement les électeurs bavarois, puisque 300000 personnes parmi les abstentionnistes, 110000 électeurs du FDP, 50000 électeurs des rangs du parti Freie Wähler Bayern, 20000 électeurs des Verts et 10000 électeurs de Die Linke ont choisi de voter pour la $\mathrm{CSU}^{(3)}$. Après le résultat catastrophique de 2008,

1 Bernd Ulrich, «Das Wählerbiest. Die Bürger sind unberechenbar und schlau - das blamiert alle Parteitaktik und zwingt zum Kampf um die Sache », www.zeitonline.de, 24. Januar 2013.

2 Michael INACKER, « Kein schöner Land ohne die FDP », www.handelsblatt.com, 20. Januar 2013.

3 Chiffres du sondage Infratest/Dimap (ARD) du 16 septembre 2013, Berechnung nach dem vorläufigen amtlichen Endergebnis. 
où le chef de gouvernement Günther Beckstein et le président du parti avaient conduit leur formation à une défaite historique, la victoire de $\mathrm{H}$. Seehofer a relégué le SPD avec ses $20,6 \%$ au second rôle. Si les Freie Wähler et les Verts ont réussi à se maintenir au niveau de $9 \%$ et 8,6\% des suffrages malgré des pertes conséquentes, les libéraux ont totalement disparu de la scène politique, confirmant la situation de fin de coalition qui s'était dessinée en Basse-Saxe et anticipant les élections fédérales à venir dans un paysage politique très particulier.

Au bout de ses cinq années passées au pouvoir à la tête de la CSU et de la Bavière, Horst Seehofer a une longue expérience de vingt-huit années de vie parlementaire et de ministères, puisqu'il fut le ministre de la Santé de Helmut Kohl et ministre de l'Agriculture de 2005 à 2008.

Partisan des compromis internes au plan local, le ministre-président bavarois occupe une position éminente parmi les dirigeants régionaux et se sert de son succès pour arriver à imposer ses vues à Berlin. Angela Merkel sait qu'elle a un partenaire exigeant. Horst Seehofer a d'ailleurs débuté la mandature en assénant certains de ses thèmes: il a évoqué un référendum auprès des adhérents pour sa propre succession en 2018, sur le modèle du SPD, alors qu'il s'était plutôt montré jusqu'alors hostile à la démocratie directe. Ce débat n'est qu'un prétexte qui lui sert naturellement à introduire la thématique dans le contexte européen dont Angela Merkel semble avoir fait son thème de prédilection pour son dernier mandat. Dans ce domaine, l'État bavarois a toujours défendu ses intérêts par un important travail de lobbying, libéral dans ses déclarations et protectionniste dans les faits. Ainsi s'est vite imposé l'exemple de la production de tabac régionale pour aller à l'encontre de la réglementation européenne en introduisant des clauses d'exception pour l'entreprise Pöschl; puis est entré dans la discussion avec les partenaires de coalition le fait d'exempter de toute obligation de salaire minimum les travailleurs saisonniers, les stagiaires ou les membres des associations ou encore les retraités complétant leurs maigres subsides par un revenu complémentaire; a été ensuite introduite la question du péage pour les automobilistes étrangers contre l'avis de larges pans de la CDU et du SPD, partenaires de coalition, qui s'est transformée en une interrogation plus large sur l'opportunité d'introduire des droits pour l'ensemble des automobilistes devant la menace autrichienne de saisir la Cour de justice européenne; enfin a surgi la polémique sur la volonté de limiter toute immigration en provenance des nouveaux arrivants de l'Union européenne, notamment Roumains et Bulgares, sur le territoire de la République fédérale, selon le slogan populiste "Celui qui triche doit partir », en utilisant subtilement la législation européenne selon laquelle tout État accueillant des travailleurs est dispensé de leur fournir des prestations sociales dans les premiers mois de leur arrivée. On pourrait dire de cette façon que la CSU inaugure la campagne européenne de l'année 2014 en mettant en avant quatre thèmes qui lui sont chers: la liberté, la sécurité, mais surtout le régionalisme et la proximité du politique avec les citoyens, jouant en cela la partition qui lui est chère en préservant les valeurs traditionnellement conservatrices de la démocratie chrétienne. Pour cela, la CSU exige une réduction du périmètre des compétences de la Commission européenne et souhaite l'introduction de la possibilité du référendum auprès des peuples sur les questions importantes touchant à ce qu'elle considère comme leur identité la plus profonde. 
En Hesse, les élections étaient concomitantes de celles qui se tenaient au plan fédéral et ont été de ce fait moins déterminantes pour le climat politique général ${ }^{(4)}$. Pendant plusieurs mois, la victoire a semblé devoir revenir au SPD et aux Verts, une grande inconnue venant toutefois des résultats du parti de gauche Die Linke et des libéraux.

Au terme d'une campagne âpre et serrée, la CDU hessoise est restée le premier parti au parlement régional. Elle atteint $42,7 \%$ des voix pour ce qui concerne les mandats directs, le ministre-président Volker Bouffier dominant symboliquement son concurrent Thorsten Schäfer-Gümbel par 46,9\% des voix contre 39,3. La CDU a cependant connu une perte de cinq circonscriptions à l'élection directe par rapport au précédent suffrage. Le FDP, partenaire de coalition, a sauvé de justesse sa présence au Landtag en atteignant le seuil des $5 \%$ des voix requises.

Le choix des électeurs est longtemps resté indécis, puisque $17 \%$ d'entre eux se sont décidés dans la phase ultime de la campagne, même si le socle le plus solide, soit $39 \%$ des électeurs de la démocratie chrétienne, avait fixé son choix depuis le départ. La remontée effectuée par la coalition au pouvoir dans les derniers mois avant l'élection s'explique par une satisfaction partagée par nombre d'électeurs envers la politique de leur gouvernement régional. La CDU a été jugée crédible sur ses compétences reconnues comme la politique économique avec $57 \%$ de satisfaction, la lutte contre la criminalité (53\% d'électeurs satisfaits) ou encore la politique de l'emploi (avec un taux d'adhésion de $50 \%$ ). Mais le fait que l'élection ait eu lieu le même jour que les élections fédérales a naturellement influencé le résultat puisque $70 \%$ des électeurs et $84 \%$ des sympathisants démocrates-chrétiens pensaient qu'Angela Merkel gouvernait bien l'Allemagne. La CDU hessoise, comme la CSU en Bavière, a su puiser les réserves nécessaires dans les rangs des abstentionnistes qui se sont ralliés à ses candidats, puisque 169000 d'entre eux se sont décidés pour elle. Autre remarque: si le parti a réuni autour de ses candidats sa clientèle provenant des rangs des professions libérales, des agriculteurs et des retraités, il a attiré à lui également en nombre les femmes âgées de 30 à 44 ans (avec une augmentation de 7,6 \% des voix), ce qui est sans doute la marque d'un large ralliement à la politique de la famille menée par la coalition au pouvoir.

À partir du début du mois de décembre 2013, des négociations ont été entamées pour former un gouvernement régional. Comme au plan national, l'un des thèmes principaux était celui de l'équité sociale, mise à mal par les réformes de Gerhard Schröder, qui sont paradoxalement la source de la bonne santé de l'économie. Volker Bouffier, le ministre-président chrétien-démocrate, et Thorsten Schäfer-Gümbel, son adversaire social-démocrate, s'étaient rencontrés à partir du 22 septembre pour tenter de mettre en place une coopération entre les deux grands partis, inspirés qu'ils étaient par ce qui se produisait au même moment au plan fédéral. Après l'impossibilité avérée de former une grande coalition avec le SPD, la tentative de ce dernier pour former une coalition de gauche aurait été une audace dans un Land plutôt conservateur et sans doute plus une posture qu'une réelle possibilité. La CDU avait quant à elle aussi décidé d'abandonner ses liens privilégiés avec le FDP, qui ne suffisait plus pour former une coalition et ne rentrait plus dans une stratégie nationale de la démocratie chrétienne

4 Sabine Pokorny, Landtagswahlen in Hessen am 22. September 2013, Wahlanalyse, Hauptabteilung Politik und Beratung, Konrad Adenauer Stiftung. 
allemande. Au terme de longs mois de pourparlers, le parti conservateur s'est donc allié à l'aile la plus réaliste des Verts, en vue de tester une stratégie qui pourrait se révéler payante en 2017. Après la tentative faite à Hambourg de 2008 à 2011, la Hesse est donc la seconde région à pratiquer ce type de coalition alliant le parti démocratechrétien aux écologistes sous la houlette de Tarek Al-Wazir. Mais l'aile la plus radicale du parti des Verts craint que cette configuration ne devienne une règle pour éviter toute coalition qui scellerait l'alliance entre les sociaux-démocrates, les écologistes et Die Linke. La Hesse pourrait devenir un modèle pour la CDU en Thuringe et en Saxe, dont les élections auront lieu à l'automne, alors que ce ne sera vraisemblablement pas le cas pour la troisième élection régionale en Brandebourg en 2014. On peut penser qu'au-delà des alliances ponctuelles à proprement parler, les accords fondamentaux sont plus profonds, notamment sur les aspects programmatiques, puisque l'un des points de convergence des partis conservateur et écologiste concerne précisément la justice intergénérationnelle. Eu égard à la question démographique, ce point est fondamental, notamment en matière de limitation des déficits, le SPD et la Gauche ayant au contraire une propension à satisfaire d'immédiates exigences. Cette alliance éloigne le spectre d'une coalition qui ferait converger la social-démocratie, le parti Die Linke et l'aile la plus fondamentaliste des Verts, garantissant à la CDU en dehors de toute considération de résultats électoraux la place de pivot de la vie politique allemande.

\section{La large victoire attendue}

Comme les sondages l'annonçaient, la victoire a bien eu lieu. Parti protéiforme représentant de la diversité régionale, la démocratie chrétienne a montré une fois de plus son adéquation avec les attentes de l'électorat allemand en conquérant 236 des 299 circonscriptions électorales de la République fédérale. Les résultats les plus remarquables pour ce qui concerne la seconde voix en faveur de la CDU sont à souligner en Bade-Wurtemberg (une augmentation de 11,2 \% du second bulletin en faveur de la $\mathrm{CDU})$, terre traditionnelle de la démocratie chrétienne malgré les succès écologiques ces dernières années, en Brandebourg (également un gain de $11,2 \%$ des voix) et en Saxe-Anhalt (là aussi une hausse de 11,1\%). La progression la plus faible de la démocratie chrétienne a été notée à Hambourg, avec seulement $4,3 \%$ de bulletins en plus en faveur de la CDU. En matière de second bulletin, les circonscriptions bavaroises ont rapporté de nombreux sièges, à l'exception de Cloppenburg-Vechta. Seuls la Rhénanie du Nord-Westphalie, la Hesse, la Basse-Saxe et le Brandebourg ont réussi à préserver quelques bastions sociaux-démocrates traditionnels, la vague démocrate-chrétienne ayant provoqué un bouleversement politique dans de nombreuses circonscriptions et provoqué un renforcement de son ancrage local. De plus, le parti conservateur a aussi progressé au-delà de la moyenne sur des territoires moins familiers comme les grandes agglomérations, excepté Duisburg. L'endroit où la démocratie chrétienne a eu ses résultats les moins probants est localisé à Berlin dans le quartier de FriedrichshainPrenzlauer Berg (15,4\%). L'électorat de la démocratie chrétienne s'est fixé relativement précocement en faveur de son orientation comme le montrent les instituts de sondage, contrairement à des échéances électorales antérieures. $37 \%$ des électeurs avaient pris leur décision depuis plusieurs mois, $18 \%$ d'entre eux réitérant leur fidélité intangible envers la CDU, alors que ce chiffre n'est que de $14 \%$ pour les autres formations. 
Plus que les suffrages acquis directement sur les candidatures personnelles, ce sont les chiffres liés à la seconde voix, c'est-à-dire au choix d'un parti, qui sont un marqueur de cette élection. La CDU a en effet obtenu un total de $41,5 \%$ des voix pour les seconds bulletins. 34,1\% ont été engrangés par la CDU et 7,4\% par la CSU. La CDU a fait un gain de 6,9\% au regard des échéances précédentes, la CSU de 0,9\%. Dans son ensemble, la démocratie chrétienne a amélioré son score de 7,7 \%. Après que les différentes composantes de la démocratie chrétienne ont obtenu 218 des 239 mandats par voie directe en 2009 grâce à ses candidats et au courant qui les portait, elle a fait encore mieux en 2013 puisqu'elle s'est arrogé directement 236 sièges au Bundestag et obtenu 75 sièges supplémentaires par l'apport du scrutin de liste. La CDU occupe ainsi 311 sièges sur 631 avec un gain de 72 députés, frisant la majorité absolue sans pourtant l'obtenir.

Mais au-delà de l'approfondissement de l'implantation régionale, c'est la sociologie du vote en faveur de la démocratie chrétienne qui semble déterminant. Elle conforte son implantation dans tous les groupes d'âge et classes sociales. Elle connaît spécifiquement une progression dans les classes d'âge les plus actives, notamment celle des 35-44 ans. La CDU n'est plus seulement le parti des couches les plus âgées de la société, mais également celui des primo-votants, puisqu'ils constituent $31 \%$ de ce segment de l'électorat. Le SPD, qui fut pendant des décennies la préférence de ce groupe d'électeurs, n'arrive qu'en seconde position avec $24 \%$ des voix malgré une légère avancée en termes électoraux.

De la même façon, la conception d'un SPD qui serait le réceptacle naturel de l'électorat féminin est aujourd'hui battue en brèche. Aujourd'hui, le parti d'Angela Merkel est le premier choix parmi l'électorat féminin, les figures antithétiques et complémentaires de la chancelière et d'Ursula von der Leyen rassemblant autour d'elles un large consensus. $44 \%$ des femmes ont opté pour la CDU, alors que ce n'est le cas que de $39 \%$ des hommes. Le SPD a suivi une pente contraire: il a séduit $27 \%$ de l'électorat masculin, contre $25 \%$ de l'électorat féminin.

\section{La démocratie chrétienne au lendemain de la victoire}

Le dernier résultat aussi faste pour la démocratie chrétienne datait de 1994. Il est sans doute le fruit de la stratégie d'Angela Merkel, qui a conduit une campagne exemplaire sans susciter de contradiction ni d'opposition. La campagne a tourné autour de sa personnalité tant l'Allemagne a semblé parfois plus se prononcer de manière référendaire envers l'action de la chancelière que de devoir voter pour une offre partisane diversifiée. Le chef d'État qu'elle est, mélange de proximité bon aloi et d'autorité internationalement reconnue, s'est placé en figure tutélaire de l'Allemagne, au-dessus de la vie partisane, pour séduire de larges pans de la population et a su mobiliser son parti pour promouvoir sa personnalité et son action plutôt que de se risquer sur une ligne programmatique hasardeuse. Le triomphe relatif de 2013 n'est pas concevable sans l'approbation et l'adhésion de nouvelles catégories d'électeurs. Outre sa réputation de sérieux et de probité irréprochable, la chancelière a profité de son aura de chef d'État ayant réagi avec sang-froid à la crise de 2008 et aux risques systémiques qui l'ont suivi.

La réélection d'un chancelier pour un troisième mandat n'est historiquement pas chose aisée si l'on considère la vie politique allemande depuis 1945. De fait, peu de chanceliers ont eu des succès aussi éclatants dans la durée, à l'exception de Konrad 
Adenauer qui avait pu améliorer son résultat en 1957, après avoir été élu brillamment en 1949 et 1953. Mais outre le caractère presque mythique de la figure qu'il représente pour la réhabilitation de la démocratie allemande, la comparaison est en effet déplacée pour ce qui concerne les partis à proprement parler. En effet, la vie politique avait connu une renaissance à l'intérieur du cadre des régions et des zones d'occupation, connaissant dans les années 1960 une concentration progressive au fur et à mesure de l'affermissement de la République fédérale d'Allemagne en tant qu'entité de référence nationale. Or la mondialisation a complètement rebattu les cartes, de sorte que l'époque est à la dilution de l'espace politique et à un éparpillement des voix qui s'agrègent autour de formations éphémères qui sont l'émanation de conjonctures et de conjonctions sociétales et thématiques, à l'instar du parti des Pirates. Contrairement à d'autres pays, les grandes formations résistent plutôt bien en Allemagne, et notamment la CDU. Par-delà le résultat immédiat, la stabilité de la démocratie chrétienne est aussi la manifestation d'une démocratie mûre, qui prouve qu'il peut exister une place pour des organisations politiques populaires dans des sociétés occidentales apaisées. Lorsqu'on associe leur score, la démocratie chrétienne et la social-démocratie brassent un spectre varié de groupes d'électeurs et restent significatives en tant que représentantes crédibles de la société allemande, avec des variations parfois étonnantes: si le SPD a ainsi obtenu des gains électoraux chez les hommes jeunes et les employés, s'il a progressé comme Die Linke chez les chômeurs, le plus grand parti des travailleurs en Allemagne reste néanmoins la CDU, contrairement à une opinion bien répandue.

\section{Angela Merkel, chancelière pour la troisième fois}

Deux fois de suite, Angela Merkel a obtenu pour la CDU/CSU un résultat qui tournait autour de $35 \%$. Elle a pu gouverner par deux fois, d'abord avec les sociaux-démocrates, puis avec les libéraux, mais le score de la démocratie chrétienne est toujours resté en deçà de ses aspirations. Ambitieuse, elle avait toujours voulu dépasser les $40 \%$ car ses grands prédécesseurs comme Konrad Adenauer, Ludwig Erhard, Kurt Georg Kiesinger, Rainer Barzel, Franz Josef Strauß et Helmut Kohl avaient déjà franchi ce cap, Edmund Stoiber faisant exception à la règle. La large victoire d'Angela Merkel la hisse au rang de ses prédécesseurs et fait de la démocratie chrétienne le seul parti populaire à part entière en ce moment en Allemagne.

Le prix en a été l'éviction des libéraux du Bundestag. Si les libéraux en sont coupables en premier lieu du fait de la médiocrité de leur personnel politique, il faut également souligner qu'Angela Merkel n'a pas voulu appeler à voter pour eux pour ce qui concerne la seconde voix. La chancelière a souhaité transformer cette adhésion exceptionnelle en une élection incontestable. Elle a voulu ce dernier succès qui lui manquait encore avant de penser à la formation d'un gouvernement. Elle ne tenait pas tant que cela au FDP comme partenaire de coalition et elle a pensé pouvoir diriger une grande coalition avec les sociaux-démocrates. C'est pour cela qu'elle a mené une campagne égocentrique et qu'elle est désormais la figure centrale de la démocratie allemande. Les $41 \%$ obtenus par la chancelière la légitiment totalement, puisque c'est elle qui a entraîné son parti vers la victoire. En effet, les contenus de la campagne électorale ont été relativement succincts. La victoire représente une césure pour la CDU. Elle a clos 
un débat, qui accompagne la chancelière depuis dix ans: c'est celui de sa modernisation et de la part de conservatisme encore possible dans un monde en mouvement.

Depuis quelques années déjà, Angela Merkel n'avait plus d'adversaire, puisqu'elle avait éliminé depuis longtemps les ministres-présidents puissants tels que Roland Koch, Christian Wulff ou Peter Müller, chaque session du présidium devenant un chemin de croix pour les impétrants en concurrence avec elle. Mais dans un passé récent, tant l'aile libérale que conservatrice avaient émis des réserves envers sa politique. La base lui a reproché de trahir le cœur de son électorat sans lequel les scores de plus de $40 \%$ ne peuvent être atteints, notamment lors des élections régionales où la CDU n'obtenait pas les scores espérés. Elle a ainsi contredit ses détracteurs, puisque la CDU et la CSU ont de nouveau retrouvé le sens de ce que signifient des partis populaires. Leurs programmes et leurs styles visaient le centre et ils se sont servis sans aucune gêne chez les sociaux-démocrates et les Verts afin de mieux les affaiblir. Angela Merkel et Horst Seehofer ont joué de concert tout en étant concurrents. Les critiques les plus acerbes ont dû se résoudre au fait qu'Angela Merkel a obtenu les résultats que les conservateurs attendaient sans reprendre un seul de leurs thèmes ou une seule de leurs exigences. Même les pertes de la démocratie chrétienne vis-à-vis de l'Alternative für Deutschland sont restées modestes, montrant que le centrisme de la chancelière n'a pas conduit à un exode des orthodoxes du parti vers d'autres formations.

\section{La grande coalition ou le choix de la CDU}

Pendant deux mois, le temps a été suspendu car la grande coalition qui se dessinait n'était pas encore en place. Chacun la souhaitait, mais comme il est de bon aloi dans une négociation, chacun faisait monter les enchères. L'un des enjeux principaux était la place du parti écologiste, qui a été résolue de manière indirecte.

La grande coalition était à la fois souhaitée par l'électorat et vraisemblablement par une grande partie de la classe politique. La chancelière, dont le pouvoir est incontesté et pour le moment incontestable, a de la sorte les moyens de faire passer les réformes les plus compliquées au Bundesrat, d'autant plus que la législature voit se profiler des conflits entre la fédération et les Länder sur des sujets cruciaux comme celui du financement de la recherche au sein des établissements universitaires. Comme il était prévu, le partenaire mineur qu'était le SPD a pris toutes les précautions avant de signer le contrat de coalition, eu égard à la première coalition sous l'égide d'Angela Merkel qui s'était soldée par une déroute cuisante pour le parti social-démocrate. Il y a eu deux grandes coalitions dans l'histoire de l'Allemagne: la première a eu lieu de 1966 à 1969 et la seconde de 2005 à 2009. La première a fait voter une réforme financière importante, puis des lois d'exception honnies en pleine période de structuration d'une opposition estudiantine. La deuxième grande coalition a eu trois phases: les débuts relativement consensuels ont permis de retarder l'âge du départ à la retraite; puis vint ensuite le temps des querelles, qui culmina en janvier 2008. Enfin, lorsque la crise éclata à l'automne 2008, les partenaires se retrouvèrent pour prendre une série de mesures : chômage partiel rémunéré à temps complet, prime à la casse et garantie pour préserver les économies des petits épargnants.

Dans une ambiance tendue, les douze groupes de travail chargés d'établir le programme du futur gouvernement ont été inventifs et productifs car la CDU et le SPD 
n'étaient pas du tout d'accord sur les largesses à distribuer à l'électorat, ayant été élus sur des programmes différents. Additionnées, leurs propositions se montaient à environ 50 milliards d'euros pour la législature, mais les négociateurs ayant refusé toute augmentation d'impôts et se faisant fort de parvenir à un budget structurellement équilibré à partir de 2014, les dépenses devaient se limiter à 15 milliards d'euros pour les quatre ans à venir, qui sont devenus 23 milliards pour arriver à mettre sur pied la coalition.

La CDU est restée sur sa ligne orthodoxe en faisant qu'il n'y ait ni d'augmentation d'impôts, ni de dérive programmée des finances publiques. En outre, les retraites de certaines mères de familles vont être revalorisées. Le SPD a obtenu l'introduction d'un salaire minimum modulable par branche et secteur, la double nationalité pour les enfants d'immigrés turcs et la possibilité pour les salariés de 63 ans de prendre leur retraite s'ils ont travaillé quarante-cinq ans et réuni leurs trimestres de cotisation. Ce dernier point était essentiel pour le SPD, qui a notamment perdu les élections de 2009 pour avoir accepté le passage progressif de la retraite à 67 ans. Quant à la CSU, elle a obtenu le droit de péage pour les étrangers sur les autoroutes, remis en question comme on l'a évoqué plus haut.

Mais il existe aussi des sujets transversaux sur lesquels se sont mis d'accord les trois partis: la sortie du nucléaire et les questions européennes. L’Allemagne respectera ses engagements de réduction d'émissions de gaz à effet de serre, mais continuera d'extraire et d'utiliser le charbon, une énergie fossile certes polluante, mais qui fait vivre des milliers de personnes en Rhénanie du Nord-Westphalie, berceau et bastion de la social-démocratie allemande. Le chapitre sur l'Europe semble davantage inspiré de la rhétorique de la CDU que de celle du SPD. Le mot compétitivité y est répété de manière récurrente, tandis que le SPD a abandonné toute perspective de mutualisation de la dette, argument pourtant souligné pendant toute la campagne. L'idée que le SPD pourrait faire éclater une grande coalition est battue en brèche par le résultat des élections. De plus, l'alternative d'une coalition avec les Verts fait peser une épée de Damoclès sur les sociaux-démocrates.

La seule question qui vaille est de savoir combien de temps Angela Merkel souhaitera se maintenir au pouvoir et ce qu'elle fera de cette troisième période de législature. Certains chanceliers ont profité de la fin de leur mandat pour prendre des décisions et des mesures ardues. Helmut Schmidt en a profité pour faire passer le réarmement de l'Allemagne, Helmut Kohl l'euro et Gerhard Schröder l'agenda 2010. Angela Merkel aime à se moquer du SPD, mais elle-même n'a jamais osé mettre en péril sa popularité avec des réformes difficiles. Il manque à la chancelière un grand dessein auquel elle pourra être associée, mais on peut imaginer qu'un approfondissement de l'unification européenne sera au cœur de ses préoccupations. Si l'on prend en compte ses déclarations et les paroles de ses conseillers, on voit les contours s'en dessiner. Jamais Angela Merkel ne s'est située, pour ce qui concerne l'Europe, du côté des plus européens de ses ministres comme U. von der Leyen, P. Altmaier ou W. Schäuble. Elle a toujours considéré Bruxelles, sans doute du fait de son éducation est-allemande, avec plus de méfiance que certains de ses collègues bercés par la chanson de geste européenne et doit aujourd'hui se confronter avec des questions qui sont en partie les conséquences des politiques successives menées par les différents chanceliers, dont son mentor Helmut Kohl. Plus d'Europe ne signifie en aucun cas pour la chancelière un renforcement 
systématique des prérogatives de la Commission européenne, car elle y voit un lieu de pouvoir loin du monde et de ses citoyens. Elle veut empêcher une élection du président de la Commission au suffrage universel bien que ce souhait ait été évoqué par la CDU dans ses contenus programmatiques, ce en quoi elle peut compter sur l'appui d'autres grandes puissances européennes. Ce que la chancelière appelle de ses vœux est une Europe de nations influentes au moyen de la modification des traités visant à renforcer le Conseil des chefs d'État européens. Ce rehaussement de l'enjeu européen sera vraisemblablement au centre de la dernière législature d'Angela Merkel, avec peut-être une réforme minimale du fédéralisme sur des bases consensuelles, et lui permettra sans doute de laisser sa trace dans l'histoire.

\section{Les nominations et la position d'Ursula von der Leyen dans la stratégie de la chancelière}

Pour ce faire, A. Merkel peut compter sur des ministres qui la connaissent et qui, par fidélité ou pour des raisons d'opportunité, lui resteront fidèles pendant toute la mandature. Il y a trois figures de proue qui sont Ursula von der Leyen, qui apparaît comme le successeur potentiel de la chancelière, Wolfgang Schäuble, l'inamovible ministre des Finances qui l'a secondée dans la tempête financière et Thomas de Maizière, son ministre de l'Intérieur fidèle d'entre les fidèles; deux ministères plus techniques sont occupés par Hermann Gröhe, qui tient les rênes de la Santé, et Johanna Wanka, ministre de l'Éducation et de la Recherche. Les trois ministres issus de la CSU viennent compléter les rangs chrétiens-démocrates: Hans-Peter Friedrich, ministre de l'Alimentation et de l'Agriculture, Alexander Dobrindt, ministre des Transports et des Réseaux numériques et Gerd Müller, ministre de la Coopération économique et du Développement.

Très paradoxalement, c'est la décision de Ronald Pofalla de quitter la direction du cabinet de la chancellerie pour rejoindre selon toute probabilité la Deutsche Bahn, qui a déterminé partiellement la réorganisation du gouvernement. En effet, peu de collaborateurs issus des rangs de la CDU étaient capables d'occuper le poste de secrétaire général de la chancellerie: Hermann Gröhe, le secrétaire général de la CDU qui avait succédé à plusieurs reprises à R. Pofalla et Peter Altmaier, a finalement été choisi. Après avoir été fonctionnaire auprès de la Commission de Bruxelles, il a été élu député en 1994 et est spécialiste des affaires de justice auprès du groupe parlementaire CDU/ CSU. Il a ensuite été nommé secrétaire d'État au ministère de l'Intérieur. En tant que chef du groupe parlementaire CDU/CSU, il a participé à l'organisation du travail gouvernemental en coopération avec le FDP.

Les quatorze ministères restants ont dû alors être répartis entre les membres de la coalition et, comme le SPD réclamait six ministères, soit un de plus que le FDP dans le précédent gouvernement, et que la CSU avait décidé d'en garder le même nombre que dans la précédente coalition, c'est-à-dire trois, il en restait cinq pour le parti d'A. Merkel, à répartir entre ses fidèles. Kristina Schröder, la précédente ministre de la Famille et de la Condition féminine, avait annoncé sa décision de partir du gouvernement. Mais le ministère n'avait que peu d'importance pour la CDU au plan stratégique. Angela Merkel souhaitant conserver Wolfgang Schäuble comme ministre des Finances, la seule question qui valait au moment de la formation du gouvernement était de savoir ce qu'il 
allait advenir d'U. von der Leyen. La première possibilité était de lui proposer le poste de ministre de la Santé, mais l'opinion publique aurait vu dans une telle nomination une sorte de désaveu. Les sondages la portent au pinacle et la ministre avait en ligne de mire bien plus que la simple campagne électorale en cours. Elle aurait bien exercé la fonction de ministre des Affaires étrangères, puisqu'elle est sans doute avec W. Schäuble la plus convaincue des Européennes. Mais depuis la première grande coalition, aucun des petits partenaires n'a jamais renoncé à ses prérogatives en la matière. Pour les ambitions d'U. von der Leyen, qui ne pouvait aspirer au ministère des Finances, il ne restait finalement que deux possibilités: le ministère de l'Intérieur ou celui de la Défense. Il existe cependant une règle tacite qui veut qu'un ministre ne soit pas obligé d'occuper un poste en adéquation avec ses compétences et sa formation, à l'exception de ceux de l'Intérieur et de la Justice où il est de bon ton que l'impétrant ait étudié le droit et obtenu le deuxième examen d'État. U. von der Leyen est médecin et A. Merkel ne voulait pour cette raison en aucun cas qu'elle prenne un poste pour lequel elle ne semblait pas qualifiée. C'est ainsi que lui est échu le poste de ministre de la Défense, compris dans toutes ses acceptions géopolitiques, c'est-à-dire comprenant des éléments de politique étrangère - à la manière dont U. von der Leyen avait exercé un droit de regard sur les droits des femmes à partir de son magistère à la tête du ministère du Travail.

Naturellement, Thomas de Maizière, dont la loyauté à la chancelière ne souffre d'aucune once de soupçon, a accepté de reprendre les rênes de son ancien ministère, Horst Seehofer l'ayant refusé au grand soulagement d'A. Merkel.

\section{Les Verts au seuil du gouvernement d'Angela Merkel}

Malgré les réticences du ministre-président de Bavière, une alliance avec les écologistes n'aurait pas semblé absurde pour la CDU. En effet, les deux formations se partagent une partie du même électorat et ne sont pas si éloignées idéologiquement. L'électorat chrétien-démocrate peut souscrire sans difficulté au principe du développement durable qui appartient au corpus fondamental des Verts. Le respect de la création au sens biblique du terme, repris dans le corpus holiste des écologistes, la responsabilité intergénérationnelle et une politique économique prenant plus en compte les PME que les grandes entreprises auraient pu être le fondement d'une coalition entre la CDU et les Verts. Même en termes de finances publiques, les deux partis sont plus proches qu'il n'y paraît puisque la CDU/CSU s'oppose à tout accroissement de l'endettement, ce qui correspond aussi au souci programmatique des Verts par égard aux générations à venir. L'assainissement des finances est la base pour les deux partis d'un État stable et réactif.

Dans la nouvelle coalition, les Verts et les députés de la Gauche siègent officiellement dans l'opposition. Pourtant, en y regardant de plus près et en considérant la manière dont fonctionne le gouvernement allemand, on observe que les écologistes ne sont jamais loin du pouvoir. En effet, dans les domaines de la justice, de l'environnement ou encore de l'économie, des secrétaires d'État issus de leurs rangs sont aux commandes de manière visible dans le nouveau gouvernement d'A. Merkel. Ils jouent un rôle de pivot important: d'un côté, les sociaux-démocrates voulaient se protéger dans des domaines aussi sensibles que la politique de la consommation, de l'énergie et de l'environnement de toute critique écologiste, de l'autre, la CDU peut apprendre à travailler avec des partenaires qui ne sont pas encore ses alliés de coalition. 
Ainsi Rainer Baake a obtenu le ressort de l'économie où il devra mettre en place la politique énergétique de la coalition. Jochen Flasbarth devra quant à lui assister la ministre de l'Environnement Barbara Hendricks et Gert Billen travaillera auprès du ministre de la Justice Heiko Maas, lui aussi social-démocrate, pour ce qui concerne le droit des consommateurs. La nomination la plus remarquée est cependant celle de Jörg Asmussen, qui a quitté la Banque centrale européenne, après avoir été secrétaire d'État sous Peer Steinbrück dans la première coalition et Wolfgang Schäuble dans la seconde, pour assister Andrea Nahles dans sa tâche. De la même façon l'écologiste Jochen Flasbarth, l'une des figures les plus éminentes du milieu écologiste, critique acerbe de la manipulation génétique, rejoint le cabinet de Barbara Hendricks.

Sans préjuger aucunement des années à venir, on peut imaginer que le mandat de la chancelière sera pour son parti celui d'un renouvellement important. Devenue la charnière de la vie politique allemande en termes stratégiques de par sa puissance et son entregent, la CDU n'a pas encore opéré sa mue programmatique. Pourtant les options qui s'ouvrent à elle en cas d'échec de la grande coalition sont réelles, à l'instar de ce qui s'est produit en Hesse. La capacité d'adaptation de la démocratie chrétienne, dont les racines s'ancrent dans les différentes constellations régionales, ne laisse aucun doute et l'on peut penser qu'au-delà des enjeux nationaux et européens, la chancelière, dont le socle de popularité ne se dément pas, aura à cœur de laisser une formation en ordre de bataille pour les échéances à venir, puisque tout laisse aujourd'hui croire que la CDU sera déterminante pour la stabilité de la démocratie allemande dans les années à venir et qu'elle continuera, à l'exception de quelques législatures passées dominées par les sociaux-démocrates, d'en rester le farouche pilier et l'inspiratrice principale.

\section{Résumé}

La CDU a connu aux élections fédérales de 2013 un succès important qui avait été largement annoncé par les instituts de sondage. Au terme d'une campagne menée sur l'argument que constituait l'image très positive d'Angela Merkel, la démocratie chrétienne a accru son ancrage dans un électorat dont les contours allaient bien au-delà de ses bases traditionnelles. Pourtant, les élections de Basse-Saxe, de Bavière et de Hesse qui avaient eu lieu la même année ont donné des résultats différents: alors que la CDU a perdu dans le premier cas en grande partie à cause de son partenaire libéral, Horst Seehofer a mis la CSU en état de partenariat fort dans la grande coalition grâce à sa large victoire aux élections régionales et Volker Bouffier est sorti en position de force de la joute électorale hessoise alors que l'issue des élections ne lui était pas obligatoirement favorable. Si la grande coalition qui s'est mise en place avec le SPD à Berlin permet de redistribuer les carte de "l'après Merkel » au sein de la CDU, elle manifeste aussi la volonté comme en Hesse de laisser la porte entrouverte pour le renouvellement des alliances avec le partenaire écologiste, dont les intérêts sociétaux et économiques convergent de plus en plus avec ceux du parti conservateur. 


\section{Zusammenfassung}

Bei den Bundestagswahlen 2013 hat die CDU einen wichtigen und von den Umfrageinstituten schon lange im Voraus prognostizierten Erfolg errungen. Nach der Wahlkampagne, die sich im Wesentlichen auf Angela Merkels positives Image bei den Wählern gestützt hat, sind die Christdemokraten noch fester in einer Wählerschaft verankert, die über ihre traditionelle Basis deutlich hinausgeht. Andererseits haben im selben Jahr die Landtagswahlen in Niedersachsen, Bayern und Hessen andere Ergebnisse produziert: Während die CDU im erstgenannten Land zum großen Teil aufgrund ihrer liberalen Partner verloren hat, konnte Horst Seehofer die CSU dank ihres großen Gewinns bei den Landtagswahlen in die Stellung eines starken Partners in der großen Koalition manövrieren, und Volker Bouffier ist gestärkt aus dem hessischen Wahlkampf hervorgegangen - obwohl das Ergebnis an sich nicht unbedingt von Vorteil für ihn war. Wenngleich die jetzt in Berlin mit der SPD geschmiedete Große Koalition es der Bundes-CDU ermöglicht, eine Neuverteilung der Karten für die Zeit „post Merkel“ vorzunehmen, zeigt sie sich (wie auch in Hessen) gleichzeitig dazu entschlossen, ein Türchen für eine Erneuerung der Zusammenarbeit mit den Grünen offen zu lassen, deren gesellschaftiche und ökonomische Konzeptionen sich denen der konservativen Partei mehr und mehr annähern. 